\title{
A Study on Satisfaction of Elderly Care Service Quality Based on Different Groups: Taking the Monitoring Data of Liaoning Province as an Example
}

\author{
Jingxing Liao*, Lei Feng, and Juxiu Huang \\ China National Institute of Standardization, China
}

\begin{abstract}
The monitoring and analysis of the social public service quality is conducive to promoting the construction of a service-oriented government that the people are satisfied with. In this paper, the third-party quality monitoring is carried out in 14 cities in Liaoning Province from 11 public service areas, including residential environment, public transportation, infrastructure, medical and health care, public security, cultural and sports, compulsory education, elderly care service, employment service, social security and administrative convenience. The results show that the public's satisfaction with social security, compulsory education, residential environment, public security and employment service is low, which needs to be paid attention to and further improved by Liaoning provincial government.
\end{abstract}

Keywords: Public Service, Quality Monitoring, Investigation and Analysis.

\section{Introduction}

With the rapid development of China's economy and society and the decisive stage of building a well-off society in an all-round way, more and more elderly people and their children are in need of high-quality elderly care service. The construction of elderly care service quality will become an important part of our country's economic development, social progress and improvement of people's livelihood for a long time in the future[1]. Providing effective high-quality elderly care service is an important direction for the development of elderly care service, and how to improve and perfect the elderly care service policy, etc. has become a key issue for the government to deal with the elderly care service quality problem[3]. In order to find out the advantages and disadvantages of the elderly care service quality, to put forward some precise suggestions, to break down development barriers through opening up "blocking points" and eliminating "pain points", and to promote the improvement of the elderly care service quality, this paper monitors the quality of elderly care services in Liaoning Province from six areas, including public elderly care services, community elderly care services, home-based elderly care services, resettlement for the

\footnotetext{
* Corresponding author: liaojx@cnis.ac.cn
} 
elderly, preferential policies and services for the elderly population, and public elderly care services[2] [4] [5].

\section{General situation of elderly care service quality in liaoning province}

The monitoring results show that in 2019, the overall evaluation level of the public satisfaction survey results of the elderly care service in Liaoning Province is "relatively satisfactory", with a score of 67.93, an increase of 0.66 compared with last year. The public's evaluation on the measurement indexes of the elderly care service is higher than that of 2018. In 2019, there are significant differences in the public's satisfaction scores in the various fields involved in the elderly care service. The public's overall feelings about the elderly care service quality and the home-based elderly care service quality score are higher, with the score of 69.33 and 69.20 , respectively. The second is the resettlement of the elderly, the preferential policies and services for the elderly, the community's elderly care service, the public elderly care service and the local elderly care service cost. The scores are 68.35, 67.86, $67.60,67.21$ and 67.19 in turn. The service quality score of local elderly care institutions is the lowest with 66.72. The scores of each index were shown in Figure 1.

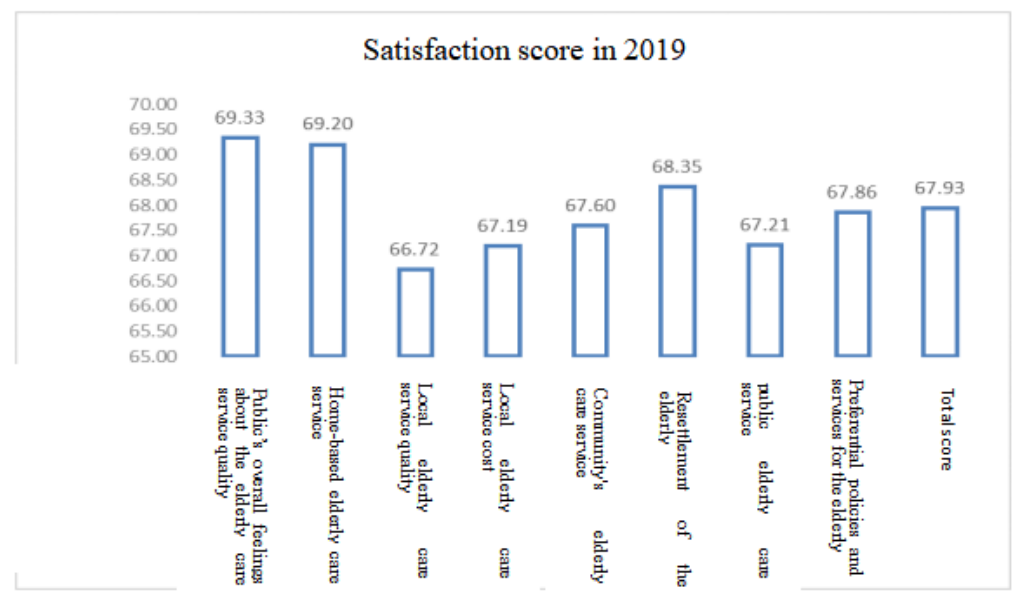

Fig.1. The public's satisfaction with the elderly care service.

\section{Analysis on the elderly care service quality in different groups}

\subsection{Quality of elderly care service in different age groups}

Statistics show that among the respondents who are willing to reveal their age in 2019 elderly care service quality satisfaction survey, there are 2,250 young people (aged 18-44), accounting for $61.78 \%$ of the total; 1,130 middle-aged people (aged 45-59), accounting for $31.03 \%$ of the total; and 262 elderly people (aged 60-79), accounting for $7.19 \%$ of the total. The data are shown in Figure 2 and Table 1. 


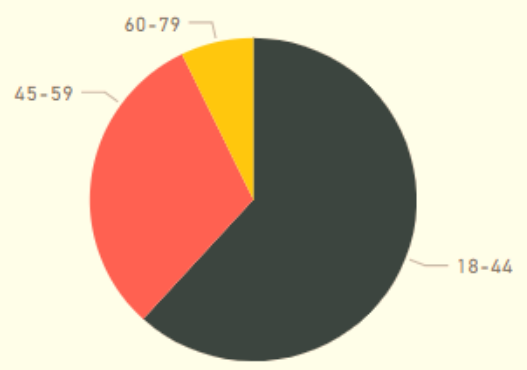

Fig. 2. The Age Proportion of the Respondents in the Social Satisfaction of the Elderly Care Service Quality in 2019

Table 1. The Age Proportion of the Respondents in the Social Satisfaction of the Elderly Care Service Quality in 2019.

\begin{tabular}{ccc}
\hline Age group & Age group count & $\%$ GT of age group count \\
\hline $18-44$ & 2250 & $61.78 \%$ \\
$45-59$ & 1130 & $31.03 \%$ \\
$60-79$ & 262 & $7.19 \%$ \\
\hline
\end{tabular}

There is a small difference in the satisfaction of the elderly care service among different age groups. Among them, the satisfaction of the elderly group and the young group to the elderly care service is higher than the overall evaluation, and the scores are 68.18 and 68.15 respectively. The satisfaction of the middle-aged group to the elderly care service is lower than the overall evaluation, which is 67.20 , as shown in Figure 3.

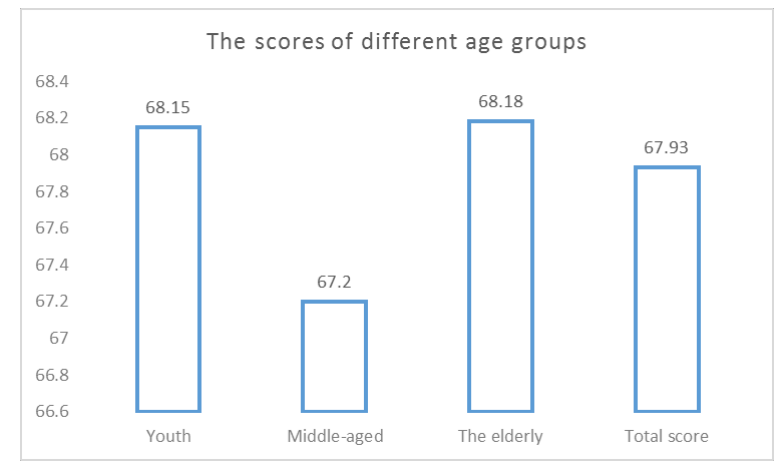

Fig. 3. Score of elderly care service satisfaction among different age groups in 2019.

\subsection{Quality of elderly care service in different gender groups}

According to the monitoring data, among the respondents who are willing to reveal their gender in the 2019 survey on the social satisfaction of the elderly care service in Liaoning Province, the number of men is 1,870 , accounting for $51.05 \%$, and that of women is 1,793 , accounting for $48.95 \%$, as shown in Table 2. 
Table 2. The sex proportion of the respondents in the elderly care service quality satisfaction in 2019.

\begin{tabular}{ccc}
\hline Gender & Gender count & \% GT of Gender count \\
\hline male & 1870 & $51.05 \%$ \\
female & 1793 & $48.95 \%$ \\
\hline
\end{tabular}

The monitoring results show that among the respondents who reveal their gender, female's satisfaction with elderly care service is higher than male's, among which female's satisfaction score is higher than the province's overall score of 68.08, and male's satisfaction is equal to the province's overall score of 67.93, as shown in Figure 4.

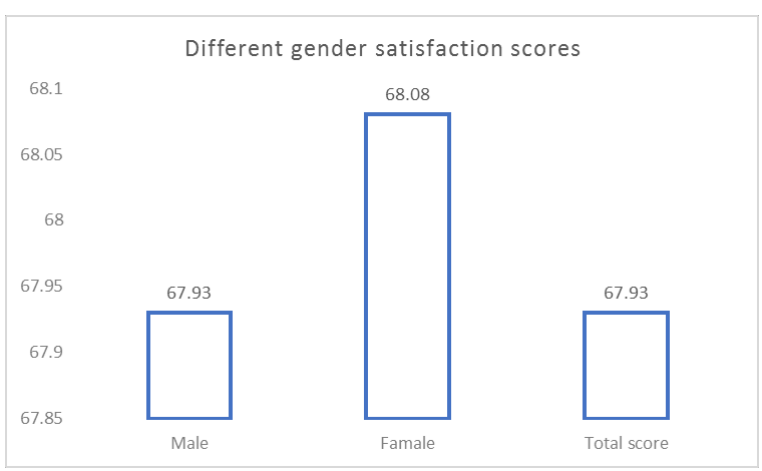

Fig. 4. Different Gender Public Satisfaction with Elderly Care Services.

\subsection{The elderly care service quality for groups with different educational levels}

According to monitoring data, among the respondents in the social satisfaction survey on the elderly care service quality in Liaoning Province in 2019, there are 158 people with primary school education, accounting for $4.30 \%$; 588 people with junior high school education, accounting for $16.01 \% ; 915$ people with senior high school / secondary school / technical school education, accounting for $24.92 \%$; 1,211 people with junior college education, accounting for $32.98 \%$, ranking first; 738 people with undergraduate education, accounting for $20.10 \%$; 62 people with master's degree or above, accounting for $1.69 \%$, ranking last, as shown in Figure 5.

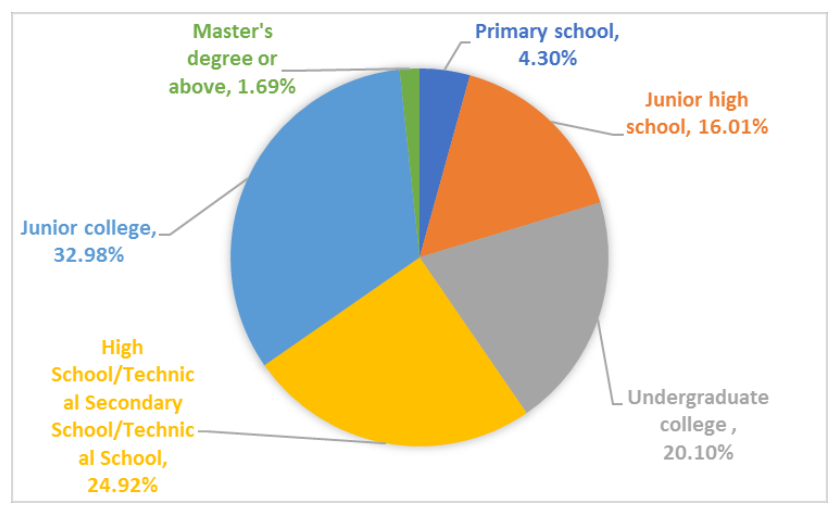

Fig. 5. The proportion of education level. 
The satisfaction of the elderly care service is different among the groups with different education levels. With the increase of the group education, the satisfaction of the elderly care service is on the rise, as shown in Figure 6.

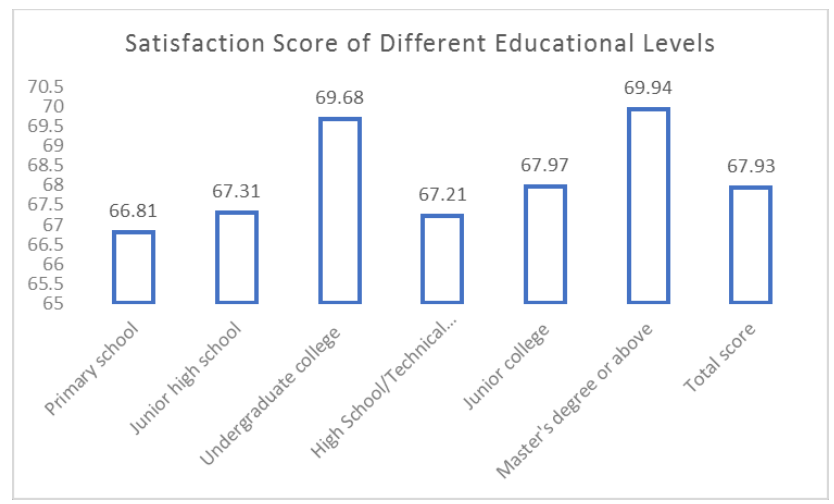

Fig. 6. Public satisfaction with elderly care services with different degrees.

\subsection{Quality of service for the aged in different income groups}

According to the statistics, among the urban and rural respondents who are willing to reveal their income in the 2019 social satisfaction survey on the elderly care service quality in Liaoning Province, the number of urban residents whose income is less than 2,000 yuan is 142 , accounting for $4.68 \%$; the number of urban residents whose income is between 2,001 and 4,000 yuan is 1,311, accounting for $43.17 \%$; the number of urban residents whose income is between 4,001 and 6,000 yuan is 1,028 , accounting for $33.85 \%$; the number of urban residents whose income is between 6,001 and 10,000 yuan is 395 , accounting for $13.01 \%$; the number of urban residents whose income is between 10,001 and 20,000 yuan is 142 , accounting for $4.68 \%$; the number of urban residents whose income is 20,001 and above is 19 , accounting for $0.63 \%$.

The number of rural residents with income less than 1,000 yuan is 27 , accounting for $4.35 \%$; the number of rural residents whose income is between 1,001 and 2,000 yuan is 74 , accounting for $11.92 \%$; the number of rural residents whose income is between 2,001 and 4,000 yuan is 343, accounting for $55.23 \%$; the number of rural residents whose income is between 4,001 and 6,000 yuan is 126 , accounting for $20.29 \%$; the number of rural residents whose income is between 6,001 and 10,000 yuan is 37 , accounting for $5.96 \%$; the number of rural residents whose income is 10,000 yuan and above is 14, accounting for $2.25 \%$. The figure is shown in Figure 7, Figure 8.

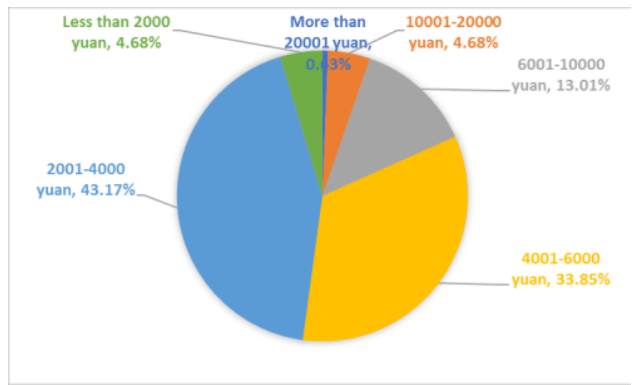

Fig.7. Urban Residents.

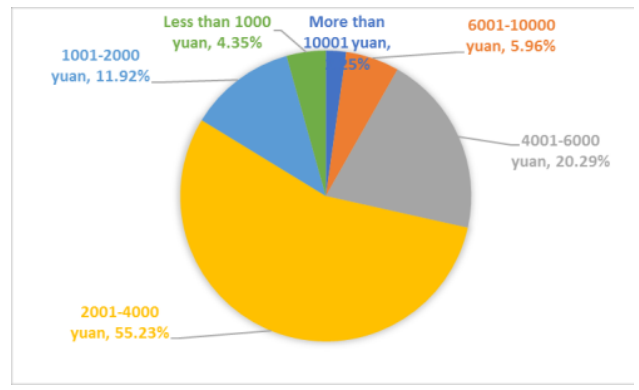

Fig. 8. Rural Residents.

The monitoring results show that the satisfaction of urban residents with the quality of elderly care service increases with the increase of average monthly family income, and the average is slightly lower than that of rural residents, indicating that the rate of improvement of elderly care service in rural areas is higher than that in urban areas. 
Specific to each family average monthly income group, the group with family average monthly income between 10,001 yuan and 20,000 yuan has the highest social satisfaction evaluation, with the score of 69.90; the group with family average monthly income above 20,000 yuan has the lower social satisfaction evaluation, with the score of 66.57 .

The rural residents' social satisfaction evaluation of the elderly care service quality basically decreases with the increase of the average monthly income of the family. Specifically, the social satisfaction evaluation of the people whose family average monthly income is less than 1,000 is higher, with the score of 71.57; the social satisfaction evaluation of the people whose family average monthly income between 4,001 yuan and 6,000 yuan is lower, with the score of 67.55. The results are shown in Figures 9 and 10.

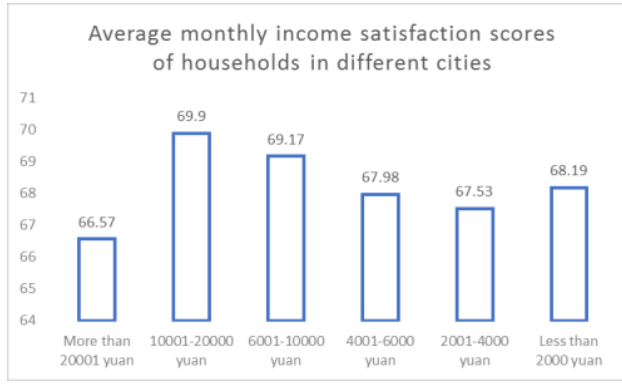

Fig.9. Income of Different Cities.

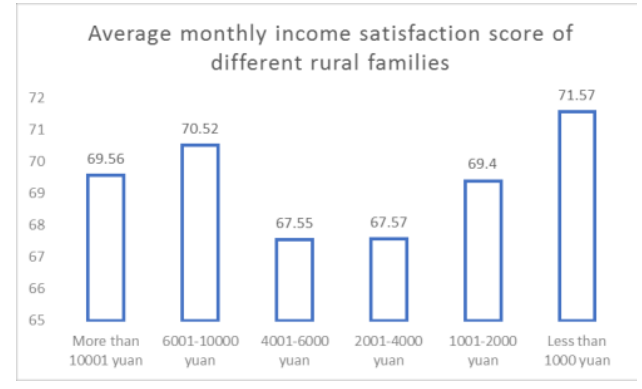

Fig. 10. Income of Different Rural.

\section{Conclusion and suggestions}

Taking the monitoring data of the elderly care service quality in Liaoning Province as an example, this paper analyzes the quality satisfaction of different groups in six fields: public elderly care service, community elderly care service, home-based elderly care service, the resettlement of the elderly, the preferential policies and services for the elderly, the public to the elderly care service and so on, and draws the following conclusions:

(1) There is little difference in the satisfaction of the elderly care service among the different age groups, and the satisfaction of the elderly care service is higher among the elderly group and the young group.

(2) Female's satisfaction with elderly care service is higher than male's.

(3) There is a great difference in the satisfaction of the elderly care service among the different education level groups, and the satisfaction of the elderly care service basically increases with the increase of the group education.

(4) The rural residents' social satisfaction evaluation of the elderly care service quality basically decreases with the increase of the average monthly income of the family. The urban residents' satisfaction evaluation of the elderly care service quality basically increases with the increase of the average monthly income of the family, and the average rate is slightly lower than that of the rural residents. The improvement rate of the elderly care service in rural areas is higher than that of the city.

In view of the above analysis results, it is suggested that according to the satisfaction of different groups, the government should carry out the targeted bench marking of the elderly care service quality, realize the precise connection between the demand and the supply of elderly care service quality, and improve the public's satisfaction with the elderly care service quality in a targeted manner so as to improve the whole level of the elderly care service quality.

\section{References}

1. D L Wang, X Z Song. China Administration. J. (6), 72-77(2009)

2. X G Luo, Hongyan Zhang. Administrative Forum. J. (3), 35-37(2008)

3. G F Cui. Hubei Social Sciences. J. (8), 37-38(2005) 
4. W B Li, L H Lai. China Administration. J.(8), 53-57(2013)

5. E'e Zhang, J Zhang. Agricultural Science Research. J.(3), 92-96(2017) 